\title{
R6 Desenvolvimento de estratégias para o Aperfeiçoamento da Produção do Conjugado Anti-IgG Canina/Peroxidase Utilizado no Kit EIE/LVC
}

Edinéa Pastro Mendes ${ }^{1}$, Hilton Jorge Nascimento ${ }^{1}$, Renata Chagas Bastos $^{1}$, Priscila Muniz da Paz', José Godinho da Silva Junior ${ }^{1}$, Maria Helena Simões Villas Bôas², Ana Paula Dinis Ano Bom ${ }^{1}$

${ }^{1}$ Bio-Manguinhos, Fiocruz, Rio de Janeiro, RJ

2 INCQS, Fiocruz, Rio de Janeiro, RJ

Introdução: Leishmaniose é uma doença endêmica presente em mais de 80 países incluindo o Brasil. A leishmaniose visceral (LV) é uma zoonose, comum ao cão e ao homem, causada pelo parasito Leishmania chagasi. O cão é considerado um importante reservatório do parasito e constitui o principal elo na cadeia de transmissão de LV. Dessa forma, o diagnóstico de LV canina (LVC) é um importante passo para evitar a transmissão da doença e a eutanásia desnecessária de cães.

Objetivo: Este estudo teve como objetivo estabelecer e aperfeiçoar estratégias para a produção de conjugados de anti-lgG canina com peroxidase (HRP) contidos no Kit de ELISA para diagnóstico da LVC (Kit EIE/LVC).

Metodologia: A anti-lgG canina foi obtida através de precipitação com sulfato de amônio, dessalinização por cromatografia de exclusão e peneiração molecular (SEC) e purificação por cromatografia de troca aniônica (IEX). A imunoglobulina purificada foi analisada por SDSPAGE, IEF-PAGE e quantificada pelo método de BCA. A conjugação da IgG à $H R P$ foi realizada pelo método de aminação redutiva, utilizando diferentes concentrações de metaperiodato de sódio e borohidreto de sódio, gerando 4 conjugados IgG/HRP distintos (1-4). A conformação dos conjugados produzidos foi avaliada por espectrometria de fluorescência e SEC. A eficiência destes conjugados foi avaliada através do ELISA contra painéis sorológicos padronizados. A estabilidade dos conjugados foi avaliada através de curvas de desnaturação térmica e testes de estabilidade acelerada (TEA), visando testar a possibilidade de armazenamento do kit no intervalo de temperatura de $2-8^{\circ} \mathrm{C}$. O TEA foi realizado em três condições de temperatura a $4^{\circ} \mathrm{C}, 37^{\circ} \mathrm{C}$ e $50^{\circ} \mathrm{C}$, utilizando o estabilizante comercial Guardian $^{\mathrm{TM}}$ em substituição ao estabilizante do kit e solução postcoating.

Resultados: A substituição da diálise por SEC e o uso da matriz de IEX Poros HQ em detrimento da matriz clássica de DEAE-Sephacel outrora usados, resultaram na diminuição do tempo de obtenção da anti-lgG canina. Após as etapas de conjugação, foi realizada a análise comparativa dos conjugados. Os experimentos de SEC indicaram que 
os conjugados apresentavam perfis cromatográficos semelhantes. 0 monitoramento da conformação dos conjugados sugere que os mesmos apresentam espectros de fluorescência semelhantes. Através do ELISA verificou-se que todos os conjugados reconheceram os soros controles positivos. Os dados de estabilidade acelerada indicaram que os conjugados se mantiveram eficientes no reconhecimento dos anticorpos, mesmo em altas temperaturas. Os conjugados apresentaram uma grande estabilidade nas análises das curvas de desnaturação térmica. Os conjugados analisados em conjunto com as placas revestidas com a solução post-coating e o estabilizante Guardian possuem estabilidade compatível para armazenamento do Kit EIE-LVC a temperatura de $2^{\circ} \mathrm{C}$ a $8^{\circ} \mathrm{C}$.

Conclusão: A implantação das novas estratégias bem como a otimização das etapas existentes na obtenção dos conjugados visa obter maior reprodutibilidade nos aspectos de produção e 0 aperfeiçoamento da qualidade do Kit EIE-LCV.

Palavras-Chave: Diagnóstico, Leishmaniose, Conjugado IgG/HRP 